\title{
Effets direct et résiduel de différents niveaux de fertilisation azotée sur la composition chimique de Brachiaria ruziziensis à la floraison à l'Ouest Cameroun
}

\author{
Fernand TENDONKENG ${ }^{1}$, Benoît BOUKILA ${ }^{2}$, Etienne T. PAMO ${ }^{1 *}$, \\ Arsène V. MBOKO ${ }^{2}$, Bienvenu F. ZOGANG ${ }^{1}$ et Férence N.E. MATUMUINI ${ }^{2}$ \\ ${ }^{I}$ Laboratoire de Nutrition Animale, Département des Productions Animales, FASA, Université de Dschang. \\ B.P. 222 Dschang, Cameroun. \\ ${ }^{2}$ Institut National Supérieur d'Agronomie et de Biotechnologie (INSAB), Université des Sciences et Techniques \\ de Masuku. B.P. 941. Gabon. \\ *Corresponding author, E-mail: ftendonkeng@yahoo.fr; pamo_te@yahoo.fr
}

\section{RESUME}

L'effet direct et résiduel de différents niveaux de fertilisation azotée sur la composition chimique de Brachiaria ruziziensis à la floraison a été réalisée à la Ferme d'Application et de Recherche de l'Université de Dschang en 2008 et 2009. Un dispositif factoriel comparant six doses d'azote $(0 ; 50 ; 100 ; 150 ; 200$ et $250 \mathrm{~kg}$ $\mathrm{N} / \mathrm{ha})$ sur des parcelles de $8 \mathrm{~m}^{2}(4 \mathrm{~m} \times 2 \mathrm{~m})$ en quatre répétitions, soit un total de 24 parcelles expérimentales a été utilisé. A la deuxième année, aucune fertilisation n'a été apportée. Un échantillon représentatif de $1 \mathrm{~kg}$ des plantes entières de B. ruziziensis a été récolté sur chaque parcelle, séparé en tiges et feuilles, puis séché à $60{ }^{\circ} \mathrm{C}$ pour l'évaluation de la composition chimique. Les résultats de ce travail ont montré que la fertilisation azotée a influencé de manière variable la composition chimique de B. ruziziensis à la floraison. Par contre, la teneur en Matières Azotées Totales (MAT) a augmenté de manière significative $(\mathrm{P}<0,05)$ avec l'effet direct de la fertilisation azotée. Les teneurs en MAT les plus élevées dans la plante entière $(5,01 \pm 0,94 \%$ MS $)$, les feuilles $(7,64 \pm 0,61 \% \mathrm{MS})$ et les tiges $(3,73 \pm 0,10 \% \mathrm{MS})$ en fonction de l'effet résiduel de la fertilisation a été obtenue respectivement avec la fertilisation aux doses 200; 0 et $250 \mathrm{~kg} \mathrm{~N} / \mathrm{ha}$. Cependant, l'effet résiduel de la fertilisation azotée n'a pas influencé de manière significative la composition chimique de la plante entière, des feuilles et des tiges de $B$. ruziziensis à ce stade phénologique. L'effet direct de la fertilisation à la floraison a entraîné une baisse de la concentration en glucides de B. ruziziensis alors qu'aucune variation significative $(\mathrm{P}>0,05)$ n'a été observée avec l'effet résiduel. Cette étude montre que l'effet direct de la fertilisation a influencé de manière significative $(\mathrm{P}<0,05)$ la teneur en protéines brutes de la plante entière, des feuilles et des tiges de B. ruziziensis alors que l'effet résiduel de la fertilisation n'a eu aucun effet significatif sur la composition chimique de la plante entière, des feuilles et des tiges à ce stade phénologique.

(C) 2011 International Formulae Group. All rights reserved.

Mots clés : $B$. ruziziensis, fertilisation azotée, composition chimique, Cameroun.

\section{INTRODUCTION}

La nécessité de nourrir une population sans cesse croissante dans les pays en voie de développement pousse de plus en plus les agriculteurs à étendre les surfaces cultivables au détriment des espaces pastoraux, ce qui a 
pour conséquence le développement des conflits entre agriculteurs et éleveurs pour l'utilisation de l'espace rural (Pamo et al., 2006). Cette situation conduit au rétrécissement des parcours naturels (Pamo et al., 2007), à les repousser vers les zones marginales et aux difficultés de satisfaire les besoins nutritionnels des animaux (Pamo et al., 2008).

Une amélioration de la productivité animale peut passer non seulement par la maîtrise des systèmes de production, mais également par l'introduction et l'intensification de la production des espèces fourragères à haut rendement. Parmi les nombreuses espèces fourragères introduites au Cameroun, la graminée pérenne Brachiaria ruziziensis, présente les avantages de mieux s'adapter dans les différentes régions, d'avoir une bonne valeur nutritive et d'être bien appétée par les animaux (Pamo et al., 2007; Tendonkeng et al., 2010). Sa culture et surtout l'intensification de son utilisation peuvent constituer un palliatif au rétrécissement des parcours ci-dessus évoqués. Les principaux facteurs influençant sa croissance, son rendement et sa valeur nutritive sont le climat, le sol et le mode d'exploitation. B. ruziziensis est proposé pour être utilisé avec profit non seulement dans l'amélioration des pâturages naturels, mais également en fourrage vert ou conservé (Cook et al., 2005). Sans fertilisation, n'importe quelle forme d'exploitation des plantes fourragères conduit à la diminution du stock en nutriments du sol en général et d'azote en particulier, surtout quand il s'agit des graminées tropicales (Obulbiga et Kabore-Zoungrana, 2007). Différentes études ont montré que la fertilisation azotée influence la composition chimique et les caractéristiques de dégradation des plantes (Peyraud et Astigarraga, 1998 ; Peyraud, 2000 ; Delaby, 2002 ; NordheimViken et Volden, 2009). Si quelques travaux ont été menés au Cameroun sur la relation entre la fertilisation et le rendement de $B$. ruziziensis (Pamo et Pieper, 1989; Pamo,
1991; Tendonkeng et al., 2010), aucun n'a encore été réalisé pour la détermination du niveau optimal de fumure azotée, et ses effets immédiats et/ou lointains sur la composition chimique de cette plante à la floraison dans les zones d'altitude.

L'objectif de cette étude est donc de déterminer l'effet direct et résiduel de différents niveaux de fumure azotée sur la composition chimique de B. ruziziensis à la floraison. De telles informations sont indispensables pour la formulation et la mise en place de meilleures stratégies de gestion de l'espèce et pour le développement de l'élevage.

\section{MATERIEL ET METHODES \\ Zone d'étude}

L'étude a été conduite à la Ferme d'Application et de Recherche (FAR) de l'Université de Dschang entre mars et novembre 2008 pour la première année, et entre mars et octobre 2009 pour la deuxième année. La FAR est située à $05^{\circ} 20^{\prime}$ latitude Nord et $10^{\circ} 03^{\prime}$ longitude Est et à une altitude moyenne de $1410 \mathrm{~m}$. Le climat de la région est équatorial de type Camerounien, modifié par l'altitude. Les températures oscillent entre $10{ }^{\circ} \mathrm{C}$ (juillet-août) et $25^{\circ} \mathrm{C}$ (février) avec une insolation annuelle de 1800 heures et une humidité relative variant entre $40-97 \%$. Les précipitations varient entre 1500 et $2000 \mathrm{~mm}$ par an. La saison sèche va de mi-novembre à mi-mars et la saison des pluies de mi-mars à mi-novembre, correspondant à la période de culture. La végétation originelle de cette région est une savane arbustive avec par endroit des forêts galeries.

\section{Dispositif expérimental}

Un dispositif factoriel en quatre répétitions à la floraison, soit un total de 24 parcelles expérimentales comparant six doses d'azote $(0 ; 50 ; 100 ; 150 ; 200$ et $250 \mathrm{~kg}$ $\mathrm{N} / \mathrm{ha})$ sous forme d'urée $(46 \mathrm{~N})$ sur des parcelles de $8 \mathrm{~m}^{2}$ (4 m x $2 \mathrm{~m}$, espacées entre elles de $0,5 \mathrm{~m}$ ) a été utilisé. Les échantillons 
du sol ont été prélevés sur le site expérimental dans l'horizon 0 - $20 \mathrm{~cm}$ avant la préparation du sol et le repiquage des éclats de souche à la première année et sur les parcelles après la fauche de régularisation à la deuxième année. L'analyse a été effectuée au Laboratoire d'Analyse des Sols, de Chimie et de l'Environnement (LABASCE) suivant la méthode d'écrite par Pauwel et al. (1992).

\section{Préparation du sol, mise en place des plants et fertilisation}

Le site expérimental a été labouré par un tracteur et la mise en place des parcelles a été faite manuellement. $80 \mathrm{~g}$ d'engrais phosphaté sous la forme de superphosphate triple a été appliquée sur toutes les parcelles comme engrais de fond. Des éclats de souche comportant trois plants de B. ruziziensis ont été prélevés dans le parcours de la FAR. Ces éclats de souche ont été habillés, et cent cinq (105) éclats de souche ont été repiqués sur chaque parcelle à $4 \mathrm{~cm}$ de profondeur, suivant un écartement de $25 \times 25 \mathrm{~cm}$.

Deux mois après la plantation d'éclats de souche, une coupe de régularisation a été effectuée à $20 \mathrm{~cm}$ au dessus du sol et les parcelles ont été fertilisées une seule fois. La deuxième année, la fauche de régulation a été effectuée en mars et sans fertilisation.

\section{Collecte des données}

Pour chaque niveau de fertilisation la première et la deuxième année, un échantillon représentatif de $1 \mathrm{~kg}$ des plantes entières a été prélevé sur les planches. Ces plantes ont été séparées en feuilles et tiges puis séchées dans une étuve à $60{ }^{\circ} \mathrm{C}$ jusqu'à poids constant. Ensuite, elles ont été broyées à l'aide d'un broyeur tri marteau aux mailles de $1 \mathrm{~mm}$ et conservés à température ambiante dans des sachets plastiques pour l'analyse de la composition chimique.

\section{Analyse de la composition chimique}

Les teneurs en matière sèche (MS), en matières azotées totales et en lipides ont été déterminées selon les méthodes décrites par AOAC (1990). Les fibres [parois cellulaires (NDF) et lignocellulose (ADF)] ont été déterminées par la méthode de Van Soest et al. (1991) sans sulfure de sodium et l' $\alpha$ amylase. La teneur en lignine-as a été déterminée en traitant l'échantillon à l'acide sulfurique $72 \%$. La teneur en glucides, la Digestibilité de la Matière Organique (DMO) et l'Energie Métabolisable (EM) ont été obtenues par les équations suivantes (Muia, 2000) :

Glucides $(\%)=$ Matière Organique (Lipides + Protéines Brutes), DMO (\%) = 91,9 - $(0,355 \times \mathrm{NDF})+(0,387 \mathrm{x}$ ADF $)-(2,17 \mathrm{x}$ Lignine-as $)$ - $(0,39 \times$ Lipides $)$ et $\mathrm{EM}=\mathrm{MOD}$ $(\%)$ x 0,$15 ; \operatorname{MOD}(\%)=(0,92 \times \mathrm{DMO})-1,2$; $\mathrm{MOD}=$ Matière Organique Digestible

\section{Analyse statistique}

Les données sur la composition chimique ont été soumises à une analyse de variance suivant le Model Linéaire Général (MLG) à l'aide du logiciel SPSS version 17.0. Lorsque les différences existaient entre les différents traitements, les moyennes étaient séparées par le test de Duncan au seuil de signification 5\% (Steel et Torrie, 1980).

\section{RESULTATS}

\section{Composition chimique du sol}

Les résultats de l'analyse du sol la première et la deuxième année sont présentés dans le Tableau 1. D'après le triangle textural (FAO), la classe texturale moyenne de ce sol est limoneuse. C'est également un sol moyennement acide $(5,4<\mathrm{pH}$-eau $<6,0)$ et ayant une acidité d'échange faible, ce qui réduit les risques de toxicité dus à un excès d'aluminium et de manganèse. La teneur moyenne en azote total de ce sol était de 3,55 $\pm 0,10 \mathrm{~g} / \mathrm{kg}$ de sol la première année et de 2,79 $\pm 0,18 \mathrm{~g} / \mathrm{kg}$ de sol la deuxième année, ce qui est suffisant pour l'agriculture traditionnelle, mais nécessite un complément azotée pour une agriculture intensive. La teneur en carbone organique était moyenne $(>2,5 \%)$ la 
première année. Il s'agit par conséquent d'un sol riche en MO (> 6\%) mais de mauvaise qualité car le rapport $\mathrm{C} / \mathrm{N}$ était supérieur à 13 (Sys et al., 1991). Par contre, à la deuxième année, le rapport $\mathrm{C} / \mathrm{N}$ était compris dans la fourchette de l'équilibre idéale (8-12) traduisant une bonne minéralisation de la matière organique (Sys et al., 1991). Pour ce qui est des bases échangeables, le sol contient des quantités moyennes de calcium, magnésium et potassium, ce qui se traduit par un rapport $\mathrm{Ca} / \mathrm{Mg}(3,24$ la première année et 3,53 la deuxième année) et $\mathrm{Mg} / \mathrm{K}(3,21$ la première année et 7,32 la deuxième année) équilibré car compris entre 1 - 5 et 3 - 15 respectivement. Par contre, la teneur moyenne en sodium de ce sol est faible $(0,27 \pm 0,06$ meq/100g), et la teneur moyenne en phosphore assimilable est très faible (1,69 \pm $0,28 \mathrm{mg} / \mathrm{kg}$ ) au cours des deux années, ce qui nécessite un apport de phosphate.

Les teneurs en bases échangeables (SBE) ont été modérées (5 - 10 méq/100g) au cours des deux années (Beernart et Bitondo, 1992). D'après Beernart et Bitondo (1992), la CEC à pH 7 serait qualifiée de faible $(<20$ méq/100g) au cours des deux années. Ces observations montrent que ce sol ne peut retenir les ions pour la nutrition des plantes, caractéristiques propres aux oxisols. Une amélioration de la CEC afin que tout engrais répandu y soit retenu pour être mis à la disposition des plantes est donc nécessaire. Pour B. ruziziensis qui exige un sol avec une fertilité élevée (Cook et al., 2005), les conditions de l'essai qui sont représentatives de celles des hautes terres de l'Ouest Cameroun sont acceptables.

Effets direct et résiduel de différents niveaux de fertilisation azotée sur la composition chimique de Brachiaria ruziziensis

Composition chimique de la plante entière

L'effet de la fertilisation azotée sur la composition chimique de la plante entière de B. ruziziensis à la floraison est présenté dans le Tableau 2. Il ressort que la teneur en MS a peu varié en fonction des traitements. La teneur en MS obtenue des plantes fauchées des parcelles non fertilisées a été significativement plus élevée $(\mathrm{p}<0,05)$ que celle obtenue des plantes fauchées des parcelles fertilisées aux doses 50, 100, 150 et $200 \mathrm{~kg} \mathrm{~N} / \mathrm{ha}$. La fertilisation a influencé de manière significative $(\mathrm{p}<0,05)$ les teneurs en cendres. Les teneurs les plus élevées ont été obtenues des plantes fauchées des parcelles fertilisées à la dose $150 \mathrm{~kg}$ N/ha $(12,57 \%$ MS). Les teneurs en cendres obtenues des plantes fauchées des parcelles fertilisées aux doses 100,150 et $250 \mathrm{~kg} \mathrm{~N} / \mathrm{ha}$ ont été significativement plus élevées que celles obtenues des plantes fauchées des parcelles non fertilisées. Les teneurs en constituants pariétaux (NDF) ont augmenté avec la dose de fertilisation azotée. Les teneurs en parois cellulaires obtenues des plantes fauchées des parcelles fertilisées aux doses 50, 100, 150 et $250 \mathrm{~kg} \mathrm{~N} / \mathrm{ha}$ ont été significativement plus élevées $(\mathrm{p}<0,05)$ que celles obtenues des plantes fauchées des parcelles non fertilisées. Les teneurs en lignocellulose ont peu varié en fonction des différents niveaux de fertilisation azotée. La valeur la plus élevée a été obtenue des plantes fauchées des parcelles fertilisées à la dose $200 \mathrm{~kg} \mathrm{~N} / \mathrm{ha}(47,18 \%$ MS et $17,09 \%$ MS) alors que la valeur la plus faible a été obtenue des plantes fauchées des parcelles non fertilisées $(45,35 \%$ MS). Cependant, aucune différence significative $(\mathrm{P}>0,05) \mathrm{n}$ 'a été obtenue entre les teneurs en lignocellulose en fonction de différents niveaux de fertilisation azotée. La DMO et l'EM ont varié significativement avec les différents niveaux de fertilisation azotée. La DMO et l'EM des plantes fauchées des parcelles non fertilisées ont été significativement $(\mathrm{P}<0,05)$ plus élevées que celles obtenues des plantes fauchées des parcelles fertilisées aux doses 200 et $250 \mathrm{~kg} \mathrm{~N} / \mathrm{ha}$. Par contre, les DMO et l'EM obtenues des plantes fauchées des parcelles fertilisées aux doses 50, 100, 150, 
200 et $250 \mathrm{~kg} \mathrm{~N} / \mathrm{ha}$ ont été comparables $(\mathrm{P}>0,05)$. La teneur en glucides de la plante entière a baissé avec l'augmentation du niveau de fertilisation azotée. Sans fertilisation, la teneur en glucides de la plante entière a été significativement $(\mathrm{P}<0,05)$ plus élevée que celle obtenue des plantes fauchées des parcelles fertilisées. Si la teneur en glucides de la plante entière fauchée des parcelles fertilisées aux doses 100,150 et $200 \mathrm{~kg} \mathrm{~N} / \mathrm{ha}$ été comparable $(\mathrm{P}>0,05)$, elle a été en revanche significativement plus élevée $(\mathrm{P}<0,05)$ que celle des plantes fertilisées à 250 $\mathrm{kg} \mathrm{N} / \mathrm{ha}$.

La deuxième année de fauche, les paramètres de la composition chimique ont varié en dents de scie en fonction l'effet résiduel résultant de différents niveaux de fertilisation azotée (Tableau 2). Cependant, aucun de ces paramètres n'a été significativement $(\mathrm{P}>0,05)$ influencé par l'effet résiduel de la fertilisation azotée.

La Figure 1 illustre l'effet direct et résiduel de la fertilisation azotée sur la teneur en MAT de la plante entière de B. ruziziensis à la floraison. Il ressort de cette figure que la teneur en MAT de la plante entière a augmenté avec le niveau de fertilisation azotée au cours de la première année de fauche. La teneur la plus faible $(9,40 \% \mathrm{MS})$ a été obtenue sur les parcelles non fertilisées tandis que la teneur la plus élevée $(12,87 \%$ MS) a été obtenue sur les parcelles fertilisées avec la plus grande dose $(250 \mathrm{~kg} \mathrm{~N} / \mathrm{ha})$. La teneur en MAT obtenue des plantes fauchées des parcelles fertilisées aux doses 50 et $100 \mathrm{~kg}$ $\mathrm{N} /$ ha a été comparable $(\mathrm{P}>0,05)$. Il en a été de même de la teneur en MAT des plantes fauchées des parcelles fertilisées aux doses 100 et $150 \mathrm{~kg} \mathrm{~N} / \mathrm{ha}$. Par contre, la fertilisation à la dose $250 \mathrm{~kg} \mathrm{~N} / \mathrm{ha}$ a permis d'obtenir une teneur en MAT dans la plante entière significativement $(p<0,05)$ plus élevée que celle obtenue avec les autres doses de fertilisation azotée à la floraison.
La deuxième année de fauche, la teneur en MAT la plus élevée $(5,01 \pm 0,92 \%$ MS) a été obtenue de l'effet résiduel de la fertilisation à la dose de $200 \mathrm{~kg} \mathrm{~N} /$ ha tandis que la teneur la plus faible $(4,32 \pm 0,49 \% \mathrm{MS})$ a été obtenue de l'effet résiduel de la fertilisation à la dose de $100 \mathrm{~kg} \mathrm{~N} / \mathrm{ha}$. Aucune différence significative $(p>0,05)$ n'a été observée entre les teneurs en MAT de la plante entière en fonction de l'effet résiduel de la fertilisation azotée.

\section{Composition chimique des feuilles}

Les résultats de l'effet direct et résiduel de différents niveaux de fertilisation sur la composition chimique des feuilles de $B$. ruziziensis à la floraison (Tableau 3) montrent que la teneur en MS a peu varié avec la fertilisation azotée. La teneur en MS des feuilles des plantes fauchées des parcelles non fertilisées a été significativement supérieure $(\mathrm{P}<0,05)$ à celle obtenue avec la fertilisation aux doses 50, 100 et $150 \mathrm{~kg} \mathrm{~N} / \mathrm{ha}$. Par ailleurs, aucune différence significative $(\mathrm{P}>0,05)$ n'a été observée entre la teneur en MS des feuilles des plantes fauchées des parcelles fertilisées aux doses 50, 100, 150, 200 et celles qui l'ont été à la dose $250 \mathrm{~kg} \mathrm{~N} / \mathrm{ha}$. Les teneurs en cendres obtenues des feuilles des plantes fauchées des parcelles non fertilisées et celles fertilisées aux doses 50 et $100 \mathrm{~kg} \mathrm{~N} /$ ha ont été comparables $(\mathrm{P}>0,05)$. La fertilisation aux doses 150, 200 et $250 \mathrm{~kg} \mathrm{~N} / \mathrm{ha}$ a permis d'obtenir des teneurs en cendres significativement $(\mathrm{P}<0,05) \quad$ supérieures à celles des plantes fauchées des parcelles non fertilisées et celles des feuilles fertilisées à la dose $50 \mathrm{~kg} \mathrm{~N} / \mathrm{ha}$. Les teneurs en constituants pariétaux (NDF) des feuilles de B. ruziziensis fauchées des parcelles non fertilisées et celles fertilisées aux doses 50, 100 et $250 \mathrm{~kg} \mathrm{~N} / \mathrm{ha}$ ont été comparables $(\mathrm{P}>0,05)$. La fertilisation aux doses 150 et $200 \mathrm{~kg} \mathrm{~N} / \mathrm{ha}$ a permis d'obtenir des constituants pariétaux significativement plus élevés $(\mathrm{P}<0,05)$ que ceux obtenus des plantes fauchées des 
parcelles non fertilisées et ceux des feuilles fertilisée à la dose $250 \mathrm{~kg} \mathrm{~N} / \mathrm{ha}$.

Les teneurs en lignocellulose ont peu varié en fonction des différents niveaux de fertilisation azotée. Les feuilles des plantes fauchées des parcelles fertilisées à la dose 150 $\mathrm{kg} \mathrm{N} / \mathrm{ha}$ ont montré des teneurs en lignocellulose plus élevées. En revanche, aucune différence significative $(P>0,05)$ n'a été observée entre les teneurs en lignocellulose des feuilles des plantes ayant reçu les autres doses. La DMO et l'EM ont varié en dents de scie. Les feuilles des plantes fauchées des parcelles fertilisées à la dose 100 $\mathrm{kg} \mathrm{N} / \mathrm{ha}$ ont eu des teneurs en DMO et EM les plus élevées $(52,34 \% \mathrm{MS}$ et 7,04 MJ/kg MS respectivement). En revanche, les feuilles des plantes fauchées des parcelles fertilisées à la dose $200 \mathrm{~kg} \mathrm{~N} / \mathrm{ha}$ ont montré des teneurs plus faibles $(48,15 \% \mathrm{MS}$ et $6,46 \mathrm{MJ} / \mathrm{kg} \mathrm{MS})$. La DMO et l'EM obtenues des feuilles des plantes fauchées des parcelles fertilisées aux doses 50,150, 200 et $250 \mathrm{~kg} \mathrm{~N} / \mathrm{ha}$ ont été comparables $(p>0,05)$. La teneur en glucide a baissé avec l'augmentation de la fertilisation. La valeur obtenue dans les feuilles des plantes fauchées des parcelles non fertilisées $(73,84 \%$ MS) a été significativement supérieure $(\mathrm{P}<0,05)$ à celle obtenue avec les autres niveaux de fertilisation. Par contre, la teneur en glucides des feuilles des plantes fauchées des parcelles fertilisées aux doses 100 et 150 $\mathrm{kg} \mathrm{N} /$ ha a été comparable ( $>0,05)$, tout comme celle des parcelles fertilisées aux doses 150 et $200 \mathrm{~kg} \mathrm{~N} / \mathrm{ha}$.

La deuxième année de fauche, les teneurs en MS, parois cellulaires, DMO, EM et glucides ont varié en dents de scie en fonction de l'effet résiduel de la fertilisation (Tableau 3). Cependant, aucun de ces paramètres n'a été significativement $(\mathrm{P}>0,05)$ influencé par l'effet résiduel de la fertilisation azotée. La teneur en cendres des feuilles des plantes fauchées des parcelles ayant reçues la fertilisation à la dose $100 \mathrm{~kg} \mathrm{~N} / \mathrm{ha}$ a été significativement plus élevée que celle obtenue des feuilles des plantes non fertilisées et celles résultant de l'effet résiduel de la fertilisation aux doses 200 et $250 \mathrm{~kg} \mathrm{~N} / \mathrm{ha}$. La teneur en lignocellulose (ADF) a varié de manière significative en fonction de l'effet résiduel de la fertilisation. Les teneurs obtenues des feuilles des plantes fauchées des parcelles non fertilisées et celles résultant de l'effet résiduel de la fertilisation à la dose de $50 \mathrm{~kg} \mathrm{~N} / \mathrm{ha}$ ont été significativement $(\mathrm{P}<0,05)$ plus élevées que celles obtenues des feuilles des plantes fauchées des parcelles ayant reçues la fertilisation à la dose de $250 \mathrm{~kg}$ N/ha. Par contre, aucune différence significative n'a été observée entre les teneurs en lignocellulose des feuilles des plantes fauchées des parcelles non fertilisées et celles résultant de l'effet résiduel de la fertilisation aux doses de 50, 100 et $250 \mathrm{~kg} \mathrm{~N} / \mathrm{ha}$. L'effet direct et résiduel de la fertilisation sur la teneur en MAT des feuilles de B. ruziziensis en fonction de la fertilisation azotée à la floraison est illustré par la Figure 2. La teneur en MAT des feuilles a augmenté avec le niveau de fertilisation azotée à la première année de fauche. Les doses d'azote 50 et 100 $\mathrm{kg} / \mathrm{ha}$ ont permis d'obtenir des teneurs en MAT comparables $(\mathrm{P}>0,05)$ dans les feuilles. Par contre, la fertilisation aux doses 50, 100, 150,200 et $250 \mathrm{~kg} \mathrm{~N} / \mathrm{ha}$ a permis d'obtenir des teneurs en MAT des feuilles, significativement $(\mathrm{P}<0,05)$ plus élevées que celles obtenues des plantes fauchées des parcelles témoins. La fertilisation à la dose $250 \mathrm{~kg} \mathrm{~N} / \mathrm{ha}$ a permis d'obtenir la teneur en MAT la plus élevée $(17,78 \%$ MS) dans les feuilles fauchées à la floraison.

La deuxième année de fauche, la teneur en MAT a peu varié en fonction de l'effet résiduel de différents niveaux de fertilisation. Les feuilles des parcelles non fertilisées ont montré des teneurs élevées en MAT (7,64 \pm $0,61 \% \mathrm{MS})$, contrairement à celles ayant été fertilisées à la dose de $250 \mathrm{~kg} \mathrm{~N} / \mathrm{ha}(6,29 \pm$ $0,41 \%$ MS). Cependant, aucune différence significative n'a été observée entre les teneurs 
en protéines brutes des feuilles de $B$. ruziziensis à ce stade phénologique en fonction de l'effet résiduel des différents niveaux de fertilisation azotée.

\section{Composition chimique des tiges}

Le Tableau 4 présente l'effet direct et résiduel de la fertilisation azotée sur la composition chimique des tiges de $B$. ruziziensis à la floraison. Son analyse montre que les teneurs en MS et cendres ont peu varié en fonction de la fertilisation à la première année de fauche. Les plantes fauchées des parcelles fertilisées à la dose $100 \mathrm{~kg}$ N/ha montrent des teneurs en cendres les plus élevées $(12,26 \%$ MS). Aucune différence significative $(\mathrm{P}>0,05)$ n'a été observée entre la teneur en MS ou la teneur en cendres en fonction de différents niveaux de fertilisation azotée. La teneur en parois cellulaires (NDF) a varié en dents de scie en fonction du niveau de la fertilisation. Contrairement aux parcelles témoins, la fertilisation à la dose $100 \mathrm{~kg} \mathrm{~N} / \mathrm{ha}$ a donné la teneur en NDF la plus élevée (74,94\% MS). Les parois cellulaires (NDF) des tiges des plantes fauchées des parcelles fertilisées aux doses 50, 100, 150, 200 et 250 $\mathrm{kg} \mathrm{N} /$ ha ont été comparables $(\mathrm{P}>0,05)$. Il en a été de même de celles des parcelles fertilisées aux doses 0, 150 et $200 \mathrm{~kg} \mathrm{~N} / \mathrm{ha}$. La teneur en lignocellulose (ADF) a peu varié en fonction du niveau de fertilisation azotée. Aucune différence significative $(p>0,05)$ n'a été observée entre les différentes valeurs obtenues selon les traitements. La DMO et l'EM ont varié en dents de scie selon la dose. Les valeurs les plus élevées en DMO et en EM ont été obtenues sur les parcelles témoins $(48,37 \%$ MS et $6,49 \mathrm{MJ} / \mathrm{kg} \mathrm{MS}$ respectivement). La fertilisation aux doses 50,100 et $250 \mathrm{~kg} \mathrm{~N} / \mathrm{ha}$ a permis d'avoir des DMO et EM comparables $(\mathrm{P}>0,05)$. La teneur en glucides des tiges a significativement $(\mathrm{P}<0,05)$ varié avec les niveaux de fertilisation azotée. Les teneurs des tiges des plantes des parcelles non fertilisées et celles fertilisées aux doses 50, 150 et $200 \mathrm{~kg} \mathrm{~N} / \mathrm{ha}$ ont été significativement supérieures $(\mathrm{P}<0,05)$ à celles des plantes des parcelles fertilisées à la dose $250 \mathrm{~kg} \mathrm{~N} / \mathrm{ha}$.

La deuxième année de fauche, les paramètres de la composition chimique des tiges de $B$. ruziziensis à ce stade phénologique ont varié en dents de scie en fonction l'effet résiduel de la fertilisation azotée (Tableau 4). Cependant, aucun de ces paramètres n'a été significativement $(\mathrm{P}>0,05)$ influencé par l'effet résiduel de la fertilisation azotée.

La Figure 3 présente l'effet direct et résiduel de la fertilisation azotée sur la teneur en MAT des tiges de B. ruziziensis à la floraison. Son analyse montre que la teneur en MAT dans les tiges à ce stade phénologique a augmenté avec les niveaux de fertilisation azotée. Les tiges des plantes fauchées des parcelles fertilisées à la dose $250 \mathrm{~kg} \mathrm{~N} / \mathrm{ha}$ ont montré des teneurs en MAT les plus élevées. Les plus faibles teneurs $(5,56 \% \mathrm{MS})$ ont été obtenues sur les parcelles non fertilisées. Les fertilisations aux doses 50, 100, 150, 200 et $250 \mathrm{~kg} \mathrm{~N} / \mathrm{ha}$ a donc permis d'augmenter de manière significative $(\mathrm{P}<0,05)$ la teneur en MAT des tiges à la floraison. La deuxième année de fauche, les teneurs en MAT obtenues des tiges des plantes fauchées des parcelles non fertilisées et celles ayant été fertilisées aux doses 50, 100 et $150 \mathrm{~kg} \mathrm{~N} /$ ha ont été comparables. Il en a été de même de celles obtenues des tiges des plantes fauchées des parcelles non fertilisées et celles ayant été fertilisées aux doses 150, 200 et $250 \mathrm{~kg} \mathrm{~N} / \mathrm{ha}$. Par contre, les teneurs en MAT obtenues des tiges des plantes fauchées des parcelles ayant été fertilisées aux doses 200 et $250 \mathrm{~kg} \mathrm{~N} / \mathrm{ha}$ ont été significativement plus élevées que celles obtenues des tiges des plantes fauchées des parcelles non fertilisées et celles ayant été fertilisées aux doses 50 et $100 \mathrm{~kg} \mathrm{~N} / \mathrm{ha}$. 


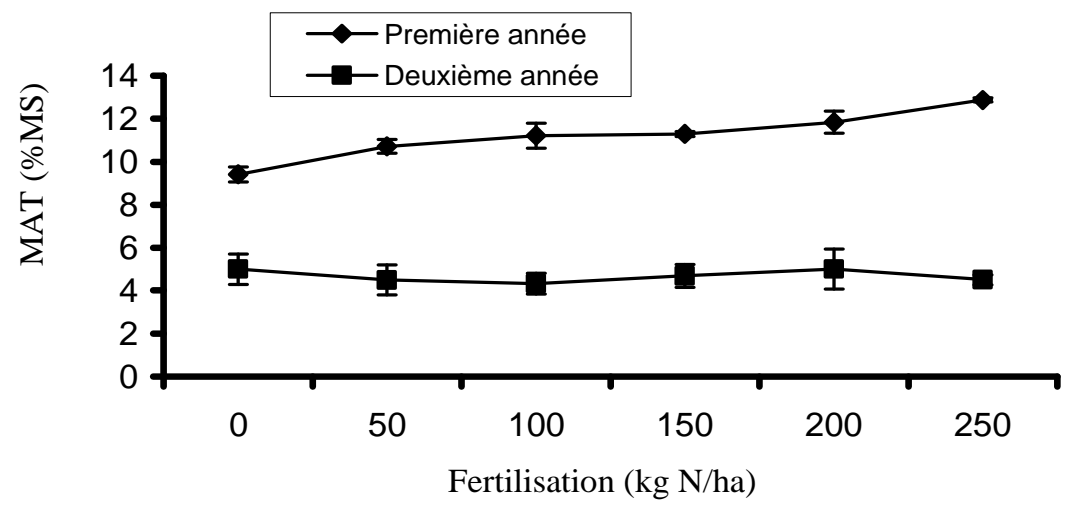

Figure 1 : Effet direct et résiduel de la fertilisation azotée sur la teneur en MAT de la plante entière à la floraison. MS : matière sèche.

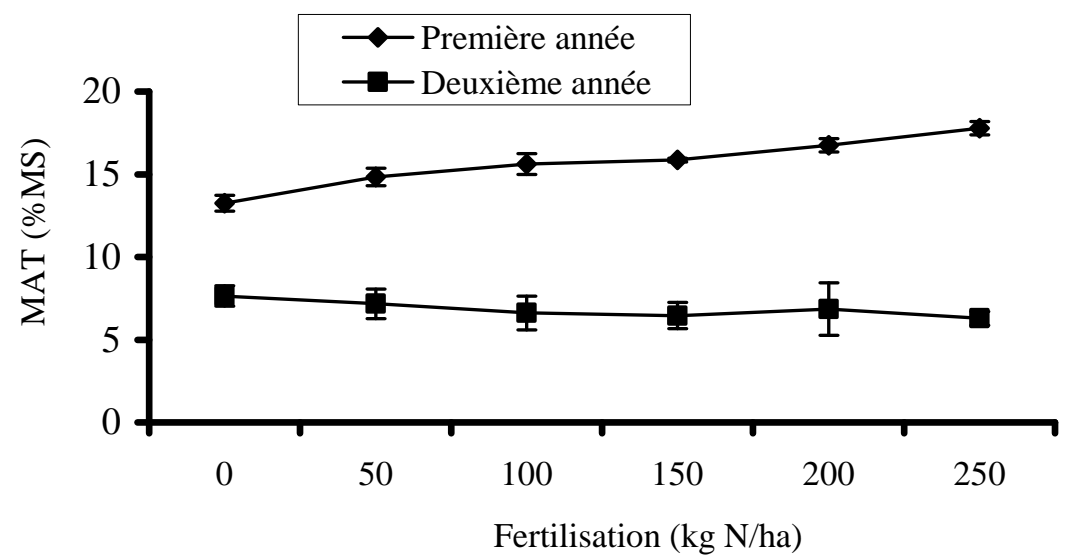

Figure 2 : Effet direct et résiduel de la fertilisation azotée sur la concentration en MAT des feuilles de Brachiaria ruziziensis à la floraison. MS : matière sèche.

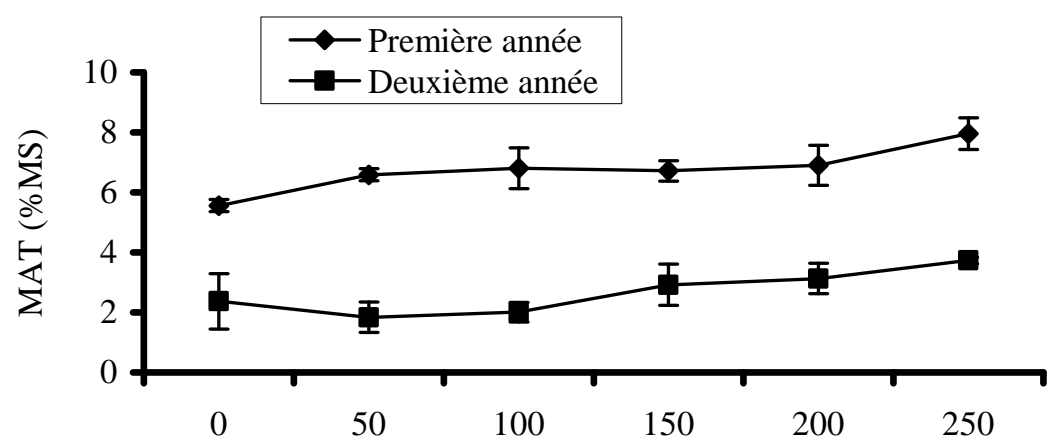

Fertilisation (kg N/ha)

Figure 3 : Effet direct et résiduel de la fertilisation azotée sur la teneur en MAT des tiges de Brachiaria ruziziensis à la floraison. MS : matière sèche. 
Tableau 1 : Analyse du sol la première et la deuxième année.

\begin{tabular}{|c|c|c|}
\hline \multirow{2}{*}{ Paramètres } & \multicolumn{2}{|c|}{ Substrat sol } \\
\hline & 2008 & 2009 \\
\hline Profondeur (\%) & $0-20$ & $0-20$ \\
\hline Pente $(\%)$ & 3 & 3 \\
\hline Horizon & Ap & Ap \\
\hline \multicolumn{3}{|l|}{ Texture (\%) } \\
\hline Sable & $14,67 \pm 2,08$ & $13,36 \pm 1,72$ \\
\hline Limon grossier & $13,67 \pm 2,08$ & $12,52 \pm 1,23$ \\
\hline Limon fin & $48,33 \pm 3,21$ & $41,87 \pm 2,51$ \\
\hline Limon total & $61,67 \pm 3,21$ & $58,37 \pm 2,45$ \\
\hline Argile & $23,67 \pm 1,15$ & $21,53 \pm 1,23$ \\
\hline Classe texturale & $\mathrm{L}$ & $\mathrm{L}$ \\
\hline \multicolumn{3}{|l|}{ Réaction du sol } \\
\hline pH-eau & $5,40 \pm 0,11$ & $5,73 \pm 0,14$ \\
\hline $\mathrm{pH}-\mathrm{KCl}$ & $4,87 \pm 0,08$ & $4,76 \pm 0,16$ \\
\hline \multicolumn{3}{|l|}{ Matière organique } \\
\hline $\mathrm{CO}(\%)$ & $4,79 \pm 0,16$ & $2,66 \pm 0,15$ \\
\hline $\mathrm{MO}(\%)$ & $8,27 \pm 0,25$ & $4,57 \pm 0,25$ \\
\hline $\mathrm{N}$ total & $3,55 \pm 0,10$ & $2,79 \pm 0,18$ \\
\hline $\mathrm{C} / \mathrm{N}$ & $13,50 \pm 0,57$ & $9,57 \pm 0,54$ \\
\hline \multicolumn{3}{|l|}{ Cations échangeables (méq/100g) } \\
\hline Calcium & $5,93 \pm 1,51$ & $6,72 \pm 1,13$ \\
\hline Magnésuim & $1,83 \pm 0,52$ & $2,49 \pm 0,73$ \\
\hline Potassium & $0,57 \pm 0,51$ & $0,34 \pm 0,07$ \\
\hline Sodium & $0,27 \pm 0,06$ & $0,10 \pm 0,01$ \\
\hline Somme des bases (SB) & $8,60 \pm 0,10$ & $9,65 \pm 1,84$ \\
\hline \multicolumn{3}{|l|}{ Capacité d'échange cationique } \\
\hline CECeff & $8,60 \pm 0,10$ & $9,65 \pm 1,84$ \\
\hline CEC à pH 7 & $19,07 \pm 4,16$ & $17,67 \pm 0,32$ \\
\hline Saturation en bases (\%) & $45,09 \pm 3,79$ & $54,61 \pm 5,34$ \\
\hline Acidité échangeable $(\mathrm{cmol}+/ \mathrm{kg})$ & $0,00 \pm 0,00$ & $0,00 \pm 0,00$ \\
\hline \multicolumn{3}{|l|}{ Phosphore du sol } \\
\hline Bray-2 (ppm ou mg/kg) & $1,69 \pm 0,28$ & $7,79 \pm 0,48$ \\
\hline
\end{tabular}


F. TENDONKENG et al. / Int. J. Biol. Chem. Sci. 5(2): 570-585, 2011

Tableau 2 : Effet direct et résiduel de la fertilisation azotée sur la composition chimique de la plante entière de Brachiaria ruziziensis à la floraison.

\begin{tabular}{|c|c|c|c|c|c|c|c|c|}
\hline \multirow{2}{*}{\multicolumn{2}{|c|}{$\begin{array}{l}\text { Fertilisation } \\
\text { (kg N/ha) }\end{array}$}} & \multicolumn{7}{|c|}{ Composition chimique } \\
\hline & & MS (\%) & $\begin{array}{l}\text { Cendres } \\
(\% \mathrm{MS})\end{array}$ & $\begin{array}{c}\text { Parois } \\
\text { cellulaires } \\
(\% \mathrm{MS})\end{array}$ & $\begin{array}{l}\text { Lignocellulose } \\
\text { (\%MS) }\end{array}$ & DMO (\%MS) & $\begin{array}{c}\text { Énergie } \\
\text { Métabolisable } \\
\text { (MJ/kg MS) }\end{array}$ & $\begin{array}{c}\text { Glucides } \\
\text { (\%MS) }\end{array}$ \\
\hline \multirow{6}{*}{$\begin{array}{l}\text { Effet } \\
\text { direct } \\
(2008)\end{array}$} & $\mathbf{0}$ & $96,83 \pm 0,51 b$ & $11,17 \pm 0,47 \mathrm{a}$ & $68,51 \pm 0,55 a$ & $45,35 \pm 0,75 a$ & $50,14 \pm 0,98 b$ & $6,74 \pm 0,13 b$ & $78,38 \pm 0,50 \mathrm{~d}$ \\
\hline & 50 & $96,10 \pm 0,27 a$ & $11,47 \pm 0,65 \mathrm{ab}$ & $70,79 \pm 1,43 b$ & $45,82 \pm 1,26 \mathrm{a}$ & $48,44 \pm 1,50 \mathrm{ab}$ & $6,50 \pm 0,21 \mathrm{ab}$ & $76,77 \pm 0,42 c$ \\
\hline & 100 & $95,81 \pm 0,51 \mathrm{a}$ & $12,42 \pm 0,64 b c$ & $71,12 \pm 1,59 \mathrm{~b}$ & $46,89 \pm 1,17 \mathrm{a}$ & $49,08 \pm 1,54 \mathrm{ab}$ & $6,59 \pm 0,21 \mathrm{ab}$ & $75,32 \pm 1,04 b$ \\
\hline & 150 & $95,84 \pm 0,34 \mathrm{a}$ & $12,57 \pm 0,88 \mathrm{c}$ & $74,10 \pm 0,68 b$ & $47,09 \pm 0,28 \mathrm{a}$ & $48,31 \pm 0,74 \mathrm{ab}$ & $6,48 \pm 0,10 \mathrm{ab}$ & $75,20 \pm 0,98 b$ \\
\hline & 200 & $96,07 \pm 0,41 \mathrm{a}$ & $12,16 \pm 0,24^{\mathrm{abc}}$ & $70,08 \pm 0,43^{\mathrm{ab}}$ & $47,18 \pm 1,23 a$ & $47,75 \pm 1,16 a$ & $6,41 \pm 0,16 \mathrm{a}$ & $74,88 \pm 0,65 b$ \\
\hline & 250 & $96,39 \pm 0,31 \mathrm{ab}$ & $12,30 \pm 0,68 \mathrm{bc}$ & $70,30 \pm 1,30 \mathrm{~b}$ & $45,93 \pm 0,87 a$ & $47,18 \pm 0,66 a$ & $6,33 \pm 0,10 \mathrm{a}$ & $72,81 \pm 0,61 \mathrm{a}$ \\
\hline \multirow{6}{*}{$\begin{array}{l}\text { Effet } \\
\text { résiduel } \\
(2009)\end{array}$} & $\mathbf{0}$ & $96,61 \pm 0,20 \mathrm{a}$ & $10,91 \pm 1,19 \mathrm{a}$ & $77,06 \pm 0,17 \mathrm{a}$ & $46,63 \pm 1,81 \mathrm{a}$ & $38,97 \pm 1,69 a$ & $5,19 \pm 0,23 a$ & $81,58 \pm 1,98 \mathrm{a}$ \\
\hline & 50 & $96,32 \pm 0,75 a$ & $11,34 \pm 1,07 \mathrm{a}$ & $77,11 \pm 2,85 a$ & $48,24 \pm 2,50 \mathrm{a}$ & $39,32 \pm 0,44 a$ & $5,24 \pm 0,06 a$ & $81,66 \pm 1,82 \mathrm{a}$ \\
\hline & 100 & $95,50 \pm 0,64 a$ & $11,20 \pm 0,61 \mathrm{a}$ & $78,17 \pm 1,66 \mathrm{a}$ & $45,85 \pm 0,65 a$ & $40,53 \pm 2,02 \mathrm{a}$ & $5,41 \pm 0,27 \mathrm{a}$ & $82,07 \pm 0,90 \mathrm{a}$ \\
\hline & 150 & $96,65 \pm 0,54 a$ & $11,18 \pm 1,31 \mathrm{a}$ & $77,77 \pm 2,29 a$ & $46,06 \pm 077 \mathrm{a}$ & $39,68 \pm 2,64 a$ & $5,20 \pm 0,36 a$ & $82,27 \pm 2,13 a$ \\
\hline & 200 & $96,32 \pm 0,32 a$ & $10,16 \pm 0,54 \mathrm{a}$ & $76,80 \pm 3,71 \mathrm{a}$ & $47,45 \pm 1,51 \mathrm{a}$ & $38,86 \pm 1,41 \mathrm{a}$ & $5,18 \pm 0,19 a$ & $82,68 \pm 1,93 a$ \\
\hline & 250 & $95,98 \pm 1,06 \mathrm{a}$ & $10,65 \pm 0,91 \mathrm{a}$ & $77,50 \pm 0,86 a$ & $45,21 \pm 2,18 \mathrm{a}$ & $37,49 \pm 0,89 a$ & $4,99 \pm 0,12 \mathrm{a}$ & $81,61 \pm 1,42 \mathrm{a}$ \\
\hline
\end{tabular}


F. TENDONKENG et al. / Int. J. Biol. Chem. Sci. 5(2): 570-585, 2011

Tableau 3 : Effet direct et résiduel de la fertilisation azotée sur la composition chimique des feuilles de Brachiaria ruziziensis à la floraison.

\begin{tabular}{|c|c|c|c|c|c|c|c|c|}
\hline \multirow[b]{2}{*}{$\begin{array}{l}\text { Fertilisation } \\
\text { (kg N/ha) }\end{array}$} & & \multicolumn{7}{|c|}{ Composition chimique } \\
\hline & & MS (\%) & $\begin{array}{l}\text { Cendres } \\
(\% \mathrm{MS})\end{array}$ & $\begin{array}{c}\text { Parois } \\
\text { cellulaires } \\
(\% \mathrm{MS})\end{array}$ & $\begin{array}{l}\text { Lignocellulose } \\
\quad(\% \mathrm{MS})\end{array}$ & DMO (\%MS) & $\begin{array}{c}\text { Energie } \\
\text { Métabolisable } \\
(\mathrm{MJ} / \mathrm{kg} \text { MS })\end{array}$ & $\begin{array}{l}\text { Glucides } \\
\text { (\%MS) }\end{array}$ \\
\hline \multirow{6}{*}{$\begin{array}{l}\text { Effet direct } \\
(2008)\end{array}$} & $\mathbf{0}$ & $96,94 \pm 0,71 b$ & $11,56 \pm 0,52 \mathrm{a}$ & $65,51 \pm 0,72 \mathrm{a}$ & $40,79 \pm 0,98 \mathrm{a}$ & $51,92 \pm 1,24 b$ & $6,98 \pm 0,17 \mathrm{~b}$ & $73,84 \pm 0,83 \mathrm{e}$ \\
\hline & 50 & $95,81 \pm 0,45 \mathrm{a}$ & $11,82 \pm 0,95 \mathrm{a}$ & $66,91 \pm 1,14 \mathrm{ab}$ & $41,08 \pm 0,95 \mathrm{a}$ & $51,47 \pm 2,60 \mathrm{ab}$ & $6,92 \pm 0,35 \mathrm{ab}$ & $71,77 \pm 0,68 d$ \\
\hline & 100 & $95,57 \pm 0,80 \mathrm{a}$ & $12,58 \pm 1,29 \mathrm{ab}$ & $67,29 \pm 1,27 \mathrm{ab}$ & $41,30 \pm 1,37 \mathrm{a}$ & $52,34 \pm 2,58 b$ & $7,04 \pm 0,35 b$ & $70,21 \pm 1,43 c$ \\
\hline & 150 & $95,48 \pm 0,39 a$ & $13,92 \pm 1,18 b$ & $68,53 \pm 0,23 b$ & $42,17 \pm 1,31 \mathrm{a}$ & $48,29 \pm 1,91 \mathrm{a}$ & $6,48 \pm 0,26 a$ & $68,95 \pm 1,11 b c$ \\
\hline & 200 & $96,14 \pm 0,47 \mathrm{ab}$ & $13,46 \pm 0,63 b$ & $67,83 \pm 0,59 b$ & $42,03 \pm 1,57 \mathrm{a}$ & $48,15 \pm 1,23 \mathrm{a}$ & $6,46 \pm 0,18 \mathrm{a}$ & $68,05 \pm 0,63 b$ \\
\hline & 250 & $96,09 \pm 0,49 \mathrm{ab}$ & $13,44 \pm 0,71 b$ & $65,82 \pm 1,80 \mathrm{a}$ & $41,24 \pm 1,25 \mathrm{a}$ & $49,47 \pm 2,14 \mathrm{ab}$ & $6,64 \pm 0,29 \mathrm{ab}$ & $66,18 \pm 0,99 a$ \\
\hline \multirow{6}{*}{$\begin{array}{l}\text { Effet résiduel } \\
(2009)\end{array}$} & $\mathbf{0}$ & $97,43 \pm 1,35 \mathrm{a}$ & $11,91 \pm 0,63 \mathrm{ab}$ & $72,27 \pm 0,59 a$ & $43,01 \pm 1,87 \mathrm{ab}$ & $45,42 \pm 3,71 \mathrm{a}$ & $6,08 \pm 0,51 \mathrm{a}$ & $78,22 \pm 1,28 \mathrm{a}$ \\
\hline & 50 & $97,93 \pm 1,38 \mathrm{a}$ & $12,90 \pm 0,87 \mathrm{bc}$ & $73,03 \pm 1,64 a$ & $46,15 \pm 3,39 c$ & $45,16 \pm 1,82 \mathrm{a}$ & $6,05 \pm 0,25 \mathrm{a}$ & $77,44 \pm 1,83 a$ \\
\hline & 100 & $95,56 \pm 0,29 a$ & $13,03 \pm 0,63 c$ & $71,71 \pm 2,32 \mathrm{a}$ & $42,07 \pm 1,95 \mathrm{abc}$ & $46,44 \pm 1,76 a$ & $6,23 \pm 0,24 a$ & $77,75 \pm 0,64 a$ \\
\hline & 150 & $97,21 \pm 0,39 a$ & $12,24 \pm 1,33^{\mathrm{abc}}$ & $73,72 \pm 2,88 \mathrm{a}$ & $40,62 \pm 0,49 \mathrm{ab}$ & $44,62 \pm 5,96 \mathrm{a}$ & $5,97 \pm 0,82 \mathrm{a}$ & $79,17 \pm 1,85 a$ \\
\hline & 200 & $96,58 \pm 0,84 a$ & $10,81 \pm 0,95 a$ & $72,05 \pm 0,64 a$ & $42,38 \pm 2,65 \mathrm{abc}$ & $44,15 \pm 2,48 \mathrm{a}$ & $5,91 \pm 0,34 \mathrm{a}$ & $79,77 \pm 1,53 a$ \\
\hline & 250 & $96,26 \pm 1,91 \mathrm{a}$ & $11,27 \pm 1,42 b$ & $72,07 \pm 1,12 \mathrm{a}$ & $38,53 \pm 3,70 \mathrm{a}$ & $41,74 \pm 0,54 \mathrm{a}$ & $5,58 \pm 0,07 \mathrm{a}$ & $78,95 \pm 1,91 \mathrm{a}$ \\
\hline
\end{tabular}


F. TENDONKENG et al. / Int. J. Biol. Chem. Sci. 5(2): 570-585, 2011

Tableau 4 : Effet direct et résiduel de la fertilisation azotée sur la composition chimique des tiges de Brachiaria ruziziensis à la floraison.

\begin{tabular}{|c|c|c|c|c|c|c|c|c|}
\hline \multirow[b]{2}{*}{$\begin{array}{l}\text { Fertilisation } \\
\text { (kg N/ha) }\end{array}$} & & \multicolumn{7}{|c|}{ Composition chimique } \\
\hline & & $\operatorname{MS}(\%)$ & $\begin{array}{l}\text { Cendres } \\
(\% \mathrm{MS})\end{array}$ & $\begin{array}{c}\text { Parois cellulaires } \\
(\% \mathrm{MS})\end{array}$ & $\begin{array}{l}\text { Lignocellulose } \\
\quad(\% \mathrm{MS})\end{array}$ & DMO (\%MS) & $\begin{array}{c}\text { Energie } \\
\text { Métabolisable } \\
(\mathrm{MJ} / \mathrm{kg} \text { MS) }\end{array}$ & Glucides (\% MS) \\
\hline \multirow{6}{*}{$\begin{array}{l}\text { Effet direct } \\
(2008)\end{array}$} & $\mathbf{0}$ & $96,73 \pm 0,84 a$ & $10,78 \pm 0,71 \mathrm{a}$ & $71,51 \pm 1,56 a$ & $49,91 \pm 0,65 a$ & $48,37 \pm 1,39 \mathrm{c}$ & $6,49 \pm 0,19 \mathrm{c}$ & $82,92 \pm 0,60 \mathrm{c}$ \\
\hline & 50 & $96,38 \pm 0,55 \mathrm{a}$ & $11,12 \pm 0,55 \mathrm{a}$ & $74,68 \pm 1,50 b$ & $50,57 \pm 1,89 a$ & $45,40 \pm 1,54 \mathrm{a}$ & $6,08 \pm 0,21 \mathrm{a}$ & $81,77 \pm 0,47 b c$ \\
\hline & 100 & $96,05 \pm 0,38 \mathrm{a}$ & $12,26 \pm 0,43 a$ & $74,94 \pm 2,51 b$ & $52,47 \pm 1,54 \mathrm{a}$ & $45,83 \pm 0,73 \mathrm{ab}$ & $6,14 \pm 0,10 \mathrm{ab}$ & $80,42 \pm 0,71 \mathrm{ab}$ \\
\hline & 150 & $96,21 \pm 0,46 a$ & $11,21 \pm 0,72 \mathrm{a}$ & $73,66 \pm 1,22 \mathrm{ab}$ & $52,02 \pm 1,38 \mathrm{a}$ & $48,33 \pm 0,73 c$ & $6,49 \pm 0,10 \mathrm{c}$ & $81,45 \pm 0,90 b$ \\
\hline & 200 & $95,99 \pm 0,70 \mathrm{a}$ & $10,86 \pm 0,64 \mathrm{a}$ & $72,32 \pm 1,15 \mathrm{ab}$ & $52,34 \pm 1,53 \mathrm{a}$ & $47,38 \pm 1,18 b c$ & $6,35 \pm 0,16 b c$ & $81,61 \pm 1,31 b c$ \\
\hline & 250 & $96,68 \pm 0,52 a$ & $11,16 \pm 1,04 \mathrm{a}$ & $74,78 \pm 1,58 b$ & $50,62 \pm 0,73 a$ & $44,89 \pm 1,02 \mathrm{a}$ & $6,01 \pm 0,14 a$ & $79,43 \pm 1,32 \mathrm{a}$ \\
\hline \multirow{6}{*}{$\begin{array}{l}\text { Effet } \\
\text { résiduel } \\
(2009)\end{array}$} & $\mathbf{0}$ & $95,79 \pm 0,15 \mathrm{a}$ & $9,80 \pm 2,29 \mathrm{a}$ & $81,94 \pm 0,35 \mathrm{a}$ & $50,25 \pm 1,77 \mathrm{a}$ & $32,52 \pm 1,35 \mathrm{a}$ & $4,31 \pm 0,18 \mathrm{a}$ & $84,94 \pm 3,16 a$ \\
\hline & $\mathbf{5 0}$ & $94,70 \pm 1,81 \mathrm{a}$ & $9,95 \pm 1,32 \mathrm{a}$ & $81,19 \pm 4,12 \mathrm{a}$ & $50,34 \pm 1,75 a$ & $33,48 \pm 1,81 \mathrm{a}$ & $4,44 \pm 0,25 \mathrm{a}$ & $85,88 \pm 1,97 \mathrm{a}$ \\
\hline & 100 & $95,44 \pm 1,13 \mathrm{a}$ & $9,38 \pm 0,99 a$ & $84,63 \pm 1,98 \mathrm{a}$ & $49,62 \pm 1,32 \mathrm{a}$ & $34,63 \pm 2,74 a$ & $4,60 \pm 0,37 a$ & $86,39 \pm 1,06 a$ \\
\hline & 150 & $96,09 \pm 0,77 \mathrm{a}$ & $10,13 \pm 1,59 a$ & $81,83 \pm 2,70 \mathrm{a}$ & $51,50 \pm 1,45 \mathrm{a}$ & $34,74 \pm 1,61 \mathrm{a}$ & $4,61 \pm 022 \mathrm{a}$ & $85,37 \pm 2,71 \mathrm{a}$ \\
\hline & 200 & $96,06 \pm 0,54 \mathrm{a}$ & $9,50 \pm 1,10 \mathrm{a}$ & $81,54 \pm 4,08 \mathrm{a}$ & $52,52 \pm 0,38 \mathrm{a}$ & $33,57 \pm 1,45 a$ & $4,45 \pm 0,20 \mathrm{a}$ & $85,59 \pm 2,47 \mathrm{a}$ \\
\hline & 250 & $95,69 \pm 0,55 a$ & $10,03 \pm 0,52 \mathrm{a}$ & $82,94 \pm 2,01 \mathrm{a}$ & $51,89 \pm 0,88 \mathrm{a}$ & $33,24 \pm 2,19 \mathrm{a}$ & $4,41 \pm 0,30 \mathrm{a}$ & $84,27 \pm 0,99 \mathrm{a}$ \\
\hline
\end{tabular}




\section{DISCUSSION}

$\mathrm{Au}$ cours de cette étude, des variations de la teneur en MS des feuilles, tiges et plante entière de $B$. ruziziensis ont été notées. Ces résultats diffèrent de ceux de Peyraud (2000) et Delaby (2000) qui font observer que classiquement, l'utilisation des quantités croissantes d'azote minéral sur les prairies de graminées pure entraîne une diminution de la teneur en matière sèche. Cette différence peut s'expliquer par l'espèce fourragère ou le type de sol. La teneur en cendres des feuilles a peu varié. Par contre, la teneur en cendres des tiges et de la plante entière de $B$. ruziziensis traitées a légèrement augmenté. Cette observation concorde avec celle de Delaby (2000) qui observe que la teneur en minéraux de l'herbe pâturée est influencée par la fertilisation azotée. En effet, l'absorption des minéraux doit s'ajuster à la vitesse d'élaboration des nouveaux tissus végétaux, c'est-à-dire la dynamique d'absorption et du métabolisme de l'azote et du carbone (Salette et Huché, 1991). Cette loi générale étant bien sûr conditionnée par la disponibilité en éléments minéraux du sol au regard de la biomasse produite sous l'effet de la fertilisation azotée (Delaby, 2000).

L'effet de la fertilisation sur les constituants pariétaux (parois cellulaires et lignocellulose) de la plante entière, des feuilles et des tiges de B. ruziziensis a été variable au cours de la première année de fauche. En effet, nous avons observé une évolution en dents de scie des teneurs en constituants pariétaux sous l'effet de la fertilisation. Ces résultats sont en accord avec ceux de Peyraud et Astigarraga (1998), Peyraud (2000), Delaby (2000) et NordheimViken et Volden (2009) qui observent que les teneurs en parois cellulaires ne sont pas affectées par la fertilisation azotée. La deuxième année de fauche, les constituants pariétaux des feuilles et de la plante entière n'ont pas été significativement influencés par l'effet résiduel de la fertilisation azotée. De même, les teneurs en parois cellulaires des feuilles ont été plus élevées à la deuxième année de fauche. Ces observations concordent avec celles de Bélanger et McQueen (1998) qui observent que le manque d'azote augmente la teneur en parois cellulaires des feuilles, mais a un moindre effet sur celles des tiges. La fertilisation azotée peut influencer de manière indirecte la teneur en parois cellulaires par des retards ou des avances de maturité (Peyraud et Astigarraga, 1998) et en modifiant le rapport feuilles/tiges (Bélanger et McQueen, 1998).

La teneur en protéines brutes dans la plante entière, les feuilles et les tiges de $B$. ruziziensis, a significativement $\quad(\mathrm{p}<0,05)$ augmenté avec l'effet direct de la fertilisation azotée. Ces observations concordent avec celles de nombreux auteurs (Peyraud et Astigarraga, 1998 ; Sarwar et al., 1999; Delaby, 2000 ; Obulbiga et Koboré Zoungrana, 2007; Nordheim-Viken et Volden, 2009; Tendonkeng et al., 2010). En effet, en absence de fertilisation azotée, la teneur en protéines brutes de l'herbe dépend d'abord et surtout de la fourniture du sol en azote (Delaby, 2000). L'augmentation de la teneur en protéines brutes de l'herbe sous l'effet de la fertilisation azotée s'accompagne d'une diminution de la part d'azote protéique au profit de l'azote non protéique (Delaby, 2000). En effet, l'entrée de l'azote dans la plante, qui s'effectue essentiellement sous forme de nitrate, s'accroît rapidement avec la fertilisation, ce qui conduit dans une première étape à l'accumulation d'azote non protéique, puis de nitrate $\left(\mathrm{N}-\mathrm{NO}_{3}\right)$ pour des niveaux de fertilisation élevée (Morot-Gaudry, 1997 ; Delaby, 2000 ; Peyraud, 2000 ; NordheimViken et Volden, 2009; Tendonkeng et al., 2010). L'azote nitrique peut alors représenter 10 à 15\% de l'azote total (Behaerge et Carlier, 1973), voire même $20 \%$ pour des fertilisations de plus de $100 \mathrm{~kg} \mathrm{~N} / \mathrm{cycle}$ (Wilman et Wright, 1978). Toutefois, la teneur en nitrate diminue rapidement avec l'âge des repousses (Peyraud, 2000). A la deuxième année de fauche, la teneur en protéines brutes de la plante entière, 
des feuilles et des tiges de $B$. ruziziensis à ce stade phénologique a varié en dents de scie en fonction de la fertilisation azotée. Ces résultats concordent avec ceux d'autres auteurs (Peyraud et Astigarraga, 1998; Sarwar et al., 1999 ; Delaby, 2000 ; Obulbiga et Koboré - Zoungrana, 2007 ; Nordheim -Viken et Volden, 2009; Nordheim - Viken et al., 2009) qui observent qu'en absence de fertilisation, la teneur en protéines brutes dans la plante dépend essentiellement de l'azote du sol.

Une baisse de la matière organique et d'énergie métabolisable a été observée en fonction de l'effet direct ou résiduel de la fertilisation azotée de la plante entière, des feuilles et des tiges de B. ruziziensis à ce stade phénologique. Ces résultats sont contraires à ceux de Peyraud et al. (1997) qui ont observé que pour des graminées récoltées au même âge, une réduction de la fertilisation azotée a peu ou pas d'effet sur la digestibilité de la matière organique. De manière générale, la teneur en glucides de B. ruziziensis au cours de la première année a significativement baissé avec la fertilisation azotée. Ces résultats ne concordent pas avec ceux de Reid et Strachan (1974) et ceux de Wilman et Wright (1978) qui ont plutôt observé un accroissement de la teneur en glucides solubles.

\section{Conclusion}

Au terme de cette étude, il ressort que : - L'effet direct de la fertilisation azotée a influencé de manière variable la composition chimique de la plante entière, des feuilles et des tiges de $B$. ruziziensis à ce stade phénologique;

- L'effet direct de la fertilisation azotée a influencé de manière significative $(\mathrm{P}<0,05)$ la teneur en protéines brutes de la plante entière, des feuilles et des tiges de $B$. ruziziensis à ce stade phénologique;

- L'effet résiduel de la fertilisation azotée n'a eu aucun effet significatif sur la composition chimique de la plante entière, des feuilles et des tiges de $B$. ruziziensis à ce stade phénologique.

\section{REFERENCES}

AOAC (Association of Official Analytical Chemist). 1991. Official Method of Analysis (15th edn). AOAC: Washington D.C.

Beernart F, Bitondo D. 1992. Simple and Practical Method to Evaluate Analytical Data of Soil Profiles. Soil Science Department. Belgian cooperation University of Dschang; 66p.

Bélanger G, McQueen RE. 1998. Analysis of the nutritive value of timothy grown with varying $\mathrm{N}$ nutrition. Grass Forage Science, 53: 109-119.

Behaeghe TJ, Carlier LA. 1973. Influence of nitrogen levels on quality and yield of herbage under mowing and grazing conditions. Proceedings of the $\mathrm{V}^{\mathrm{th}}$ General Meeting of European Grassland Federation, Uppsala, pp. 52-66.

Cook BG, Pengelly BC, Brown SD, Donnelly JL, Eagles DA, Franco MA, Hanson J, Mullen BF, Partridge IJ, Peters M, Schultze-Kraft R. 2005. Tropical Forages: an Interactive Selection Tool. [CD-ROM], CSIRO, DPI\&F(QId), CIAT and ILRI: Brisbane, Australia.

Delaby L. 2000. Effect of mineral nitrogen fertilization on the feeding value of herbage and the performance of grazing dairy cows. Fourrages, 164: 421-436.

Euroconsult 1989. Agricultural Compedium for Rural Development in the Tropics and Subtropics. Elsevier: Amsterdam; 740 p.

Lawlor D, Lemaire G, Gastal F. 2001. Nitrogen, plant growth and crop yield. In Plant Nitrogen, Lea PJ, Morot-Gaudry JF (eds). INRA; 343-367.

Morot-Gaudry J-F. 1997. Assimilation de l'Azote chez les Plantes: Aspects Physiologique, Biochimique et Moléculaire. Edition INRA; 422p.

Muia JMK. 2000. Use of Napier grass to improve smallholder milk production in 
Kenya. PhD Thesis, Wageningen Agricultural University, The Netherlands.

Nordheim-Viken H, Volden H. 2009. Effect of maturity stage, nitrogen fertilization and seasonal variation on ruminal degradation characteristics of neutral detergent fibre in timothy (Phleum pratense L.). Anim. Feed Sci. Technol., 149: 30-59.

Nordheim-Viken H, Volden H, Jørgensen M. 2009. Effects of maturity stage, temperature and photoperiod on growth and nutritive value of timothy (Phleum pratense L.). Anim. Feed Sci. Technol., 152: 204-218.

Obulbiga MF, Kaboré-Zoungrana CY. 2007. Influence de la fumure azotée et du rythme d'exploitation sur la production de matière sèche et la valeur alimentaire de Andropogon gayanus kunth au Burkina Faso. Tropicultura, 25(3): 161 167.

Pamo TE, Boukila B, Tendonkeng F. 2007. Goat production in Africa: a sign post review for research in the new millennium. Int. J. Biol. Chem. Sci., 1(1): 76-89.

Pamo TE, Boukila B, Meduke CN, Tendonkeng F. 2008. Effect of nitrogen fertilisation and cutting frequency on the yield and regrowth of Panicum maximum Jacq in West Cameroon. XXI International Grassland Congress / VIII International Rangeland Congress, Hohhot, China, $29^{\text {th }}$ June $-5^{\text {th }}$ July 2008. p 354.

Pamo TE, Fonteh FA, Tendonkeng F, Kana JR, Djaga PJ, Fomewang II G. 2006. Influence of supplementary feeding of multipurpose leguminous tree leaves on kid growth and milk production of the West African Dwarf goats. Small Rum. Res., 63: 142-149.

Pamo TE, Pieper RD. 1989. Effect of nitrogen fertilization in combination with potassium and phosphorus and cutting frequency on the yield of Brachiaria ruziziensis in Adamawa plateau, Cameroon. XVI International Grassland congress, 4-11 october 1989, Nice France. Versailles, The French Grassland society, 1989. p. 111-112.

Pamo TE. 1991. Réponse de Brachiaria ruziziensis Germain et Evard à la fertilisation azotée et à différents rythmes d'exploitation en Adamaoua, Cameroun. Rev. Elev. Méd. Vét. Pays Trop., 44(3): $373-380$.

Pauwels JM, Van Ranst E, Verloo M, Mvondo Ze A. 1992. Méthode d'Analyse de Sols et de Plantes, Gestion de Stock de Verrerie et de Produits Chimiques. Manuel de Laboratoire de Pédologie. Publication Agricoles; 286p.

Peyraud JL. 2000. Fertilisation azotée des prairies et nutrition des vaches laitières. Conséquences sur les rejets d'azote. INRA. Prod. Anim., 13(147 - 188).

Peyraud JL, Astigarraga L. 1998. Review of the effect of nitrogen fertilization on the chemical composition, intake, digestion and nutritive value of fresh herbage: consequences on animal nutrition and $\mathrm{N}$ balance. Anim. feed Sci. Technol., 72: 235-259.

Peyraud JL, Astigarraga L, Faverdin P. 1997. Digestion of fresh perennial ryegrass fertilised at two levels of nitrogen by lactating dairy cows. Anim. Feed Sci. Technol., 64: 155-171.

Reid RL. Strachan NH. 1974. The effects of a wide range of nitrogen rates on some chemical constituents of the herbage from perennial ryegrass swards with and without white clover. J. Agric. Sci., 83: 393-401.

Sarwar M, Khan, Mahr-un-Nisa, Saeed M.N. 1999. Influence of nitrogen fertilization and stage of maturity of mottgrass (Pennisetum purpureum) on its composition, dry matter intake, ruminal characteristics and digestion kinetics in cannulated buffalo bulls. Anim. Feed Sci. Technol., 82: 121-130. 
Salette J, Huché L. 1991. Diagnostic de l'état de nutrition minérale d'une prairie par l'analyse du végétal: Principe, mise en œuvre. Fourrages, 125 : 3-18.

Steel RG, Torrie JH. 1980. Principles and Procedures of Statistics. McGraw Hill Book C: New York; 633p.

Sys C, Van Ranst E, Debaveye J, Beernaert F. 1991. Land Evaluation, part II. Methods for Land Evaluation. Belgium, General Administration for Development Cooperation.

Tendonkeng F, Boukila B, Pamo TE, Mboko AV, Tchoumboué J. 2010. Effet de différents niveaux de fertilisation azotée sur le rendement et la composition chimique de Brachiaria ruziziensis à la montaison dans l'Ouest Cameroun. Livestock Research for Rural Development. Volume 22, Article \#19. Retrieved December 13, 2010, from http://www.lrrd.org/lrrd22/1/tend22019.ht $\mathrm{m}$

Van Soest JP, Robertson JB, Lewis BA. 1991. Methods for dietary fibre, neutral detergent fibre and non-starch polysaccharides in relation to animal production. J. Dairy Sci., 74: 3583-3597.

Wilman D, Wright PT. 1978. The proportions of cell content, nitrogen, nitrate-nitrogen and water-soluble carbohydrates of three grasses in early stages of regrowth after defoliation with or without applied nitrogen. J. Agric. Sci., 91: 381-394. 